WellBeing International

WBI Studies Repository

$2-2006$

\title{
Animal Carcinogenicity Studies: 2. Obstacles to Extrapolation of Data to Humans
}

\author{
Andrew Knight \\ Animal Consultants International \\ Jarrod Bailey \\ University of Newcastle-upon-Tyne \\ Jonathan Balcombe \\ Independent Scientist and Author
}

Follow this and additional works at: https://www.wellbeingintlstudiesrepository.org/acwp_arte

Part of the Animal Experimentation and Research Commons, Animal Studies Commons, and the Other Medical Sciences Commons

\section{Recommended Citation}

Knight, A., Bailey, J., \& Balcombe, J. (2006). Animal carcinogenicity studies: 2. Obstacles to Extrapolation of Data to Humans. ATLA-NOTTINGHAM-, 34(1), 29.

This material is brought to you for free and open access by WellBeing International. It has been accepted for inclusion by an authorized administrator of the WBI Studies Repository. For more information, please contact wbisr-info@wellbeingintl.org.

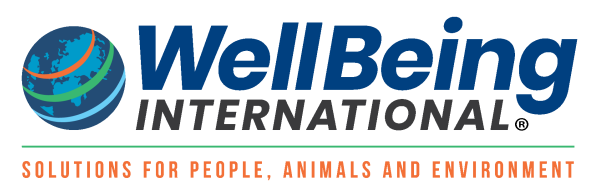




\title{
Animal Carcinogenicity Studies: 2. Obstacles to Extrapolation of Data to Humans
}

\author{
Andrew Knight, ${ }^{1}$ Jarrod Bailey² and Jonathan Balcombe ${ }^{3}$ \\ ${ }^{1}$ Animal Consultants International, London, UK; 2 School of Population and Health Sciences, Faculty of \\ Medical Sciences, University of Newcastle upon Tyne, Newcastle upon Tyne, UK; 3Physicians Committee for \\ Responsible Medicine, Washington DC, USA
}

\begin{abstract}
Summary - Due to limited human exposure data, risk classification and the consequent regulation of exposure to potential carcinogens has conventionally relied mainly upon animal tests. However, several investigations have revealed animal carcinogenicity data to be lacking in human predictivity. To investigate the reasons for this, we surveyed 160 chemicals possessing animal but not human exposure data within the US Environmental Protection Agency chemicals database, but which had received human carcinogenicity assessments by 1 January 2004. We discovered the use of a wide variety of species, with rodents predominating, and of a wide variety of routes of administration, and that there were effects on a particularly wide variety of organ systems. The likely causes of the poor human predictivity of rodent carcinogenicity bioassays include: 1) the profound discordance of bioassay results between rodent species, strains and genders, and further, between rodents and human beings; 2) the variable, yet substantial, stresses caused by handling and restraint, and the stressful routes of administration common to carcinogenicity bioassays, and their effects on hormonal regulation, immune status and predisposition to carcinogenesis; 3) differences in rates of absorption and transport mechanisms between test routes of administration and other important human routes of exposure; 4) the considerable variability of organ systems in response to carcinogenic insults, both between and within species; and 5) the predisposition of chronic high dose bioassays toward false positive results, due to the overwhelming of physiological defences, and the unnatural elevation of cell division rates during ad libitum feeding studies. Such factors render profoundly difficult any attempts to accurately extrapolate human carcinogenic hazards from animal data.
\end{abstract}

Key words: animal experiment, animal test, bioassay, cancer prevention, carcinogenicity, chemical classification, chemical safety, extrapolation, risk assessment.

Address for correspondence: A. Knight, Animal Consultants International, 91 Vanbrugh Court, Wincott Street, London SE11 4NR, UK.

E-mail: info@animalconsultants.org

\section{Introduction}

Due to a paucity of human exposure data, public health policy and the regulation of exposures to potential human carcinogens by governmental agencies such as the US Environmental Protection Agency (EPA) have for decades relied mainly upon animal carcinogenicity tests. However, our survey of 160 environmental contaminants considered to be of greatest concern within the USA, listed in the EPA's Integrated Risk Information System (IRIS) chemicals database, that lacked significant human exposure data but possessed animal data, found that the EPA considered the animal data inadequate to support a classification of probable human carcinogen or non-carcinogen in the majority (58.1\%; 93/160) of cases (1). Significant differences in human carcinogenicity classifications of identical chemicals between the EPA and the World Health Organisation's International Agency for Research on Cancer (IARC) indicate that the true predictivity for human carcinogenicity of animal data is even less than that indicated by EPA figures alone.
The sensitivity of the conventional rodent bioassay to human carcinogens (the ability to detect them) for some sex-species combinations is not in question. However, it's very poor human specificity (the ability to identify human non-carcinogens) has also been revealed by other investigators (2-6), and contributes to the poor human predictivity of the assay, that is, the low probability that a positive result is truly indicative of human carcinogenicity. To investigate the reasons for the poor human specificity and predictivity of animal carcinogenicity tests, we examined the IRIS chemicals that had received EPA human carcinogenicity assessments primarily on the basis of animal carcinogenicity data.

\section{Methods}

Of the 543 chemicals contained in the IRIS chemicals database as of 1 January 2004, 160 lacked significant human exposure data, but possessed animal data and had received human carcinogenicity assessments. For each of these 160 chemicals, 
we determined the species and routes of administration used, and the organ systems affected.

\section{Results}

\section{Species used}

The species used were not provided for two of the 160 chemicals, both of which were considered unclassifiable as to their human carcinogenicity. The other 158 chemicals were each studied in up to seven different species (in the case of $\mathrm{N}$-nitrosodiethylamine), with the most common numbers of species used per chemical (Table 1) being two $(67.7 \%)$, one $(14.6 \%$, many, but not all of which resulted in a human carcinogenicity assessment of "unclassifiable"), and three (11.4\%).

At least 10 different species were used for these 158 chemicals, namely: chickens, dogs, guinea-pigs, hamsters, mice, mink, primates (one macaque, three unspecified "monkey" species, and one unspecified "primate" species), rabbits, rats and trout. The three species most commonly used per chemical (Figure 1) were mice $(92.4 \%)$, rats $(86.7 \%)$ and hamsters $(14.6 \%)$.

\section{Routes of administration}

Routes of administration were not provided for four of the 160 chemicals. Up to 10 routes of administration (in the case of benzo[a]pyrene) were used for each of the other 156 chemicals, with the most common numbers of routes used per chemical (Table 2) being one (43.6\%), two (21.1\%) and three (19.2\%).

Table 1: Species used with assessed EPA chemicals lacking significant human data but possessing animal data

\begin{tabular}{lcc}
\hline $\begin{array}{l}\text { Number of } \\
\text { species used }\end{array}$ & $\begin{array}{c}\text { Number of } \\
\text { chemicals }\end{array}$ & $\begin{array}{c}\text { \% of total } \\
\text { chemicals }\end{array}$ \\
\hline 1 & 23 & 14.6 \\
2 & 107 & 67.7 \\
3 & 18 & 11.4 \\
4 & 6 & 3.8 \\
5 & 0 & 0.0 \\
6 & 3 & 1.9 \\
7 & 1 & 0.6 \\
\hline Total & 158 & \\
\hline
\end{tabular}

Data source: The EPA Integrated Risk Information System database, 1 January 2004.
Table 2: Routes of administration for assessed EPA chemicals lacking significant human data but possessing animal data

\begin{tabular}{lcc}
\hline $\begin{array}{l}\text { Number of routes } \\
\text { of administration }\end{array}$ & $\begin{array}{c}\text { Number of } \\
\text { chemicals }\end{array}$ & $\begin{array}{c}\text { \% of total } \\
\text { chemicals }\end{array}$ \\
\hline 1 & 68 & 43.6 \\
2 & 33 & 21.1 \\
3 & 30 & 19.2 \\
4 & 16 & 10.3 \\
5 & 4 & 2.6 \\
6 & 4 & 2.6 \\
10 & 1 & 0.6 \\
\hline Total & 156 \\
\hline
\end{tabular}

Data source: The EPA Integrated Risk Information System database, 1 January 2004.

Twelve non-oral routes of administration and a variety of oral routes, not always specified, were used (Figure 2), namely: dermal, inhalation, intramuscular, intraperitoneal, intrapleural, intrarenal, intratesticular, intravenous, oral (food), oral (gavage), oral (water), oral (other, e.g. capsule, toothpaste additive), oral (unspecified), subcutaneous, surgical implantation, transplacental, and vaginal painting. Those most commonly used were food $(49.4 \%)$, gavage $(33.3 \%)$ and dermal administration

Table 3: Organ systems affected by chemicals assessed by the EPA to be probable (B2) or possible (C) human carcinogens

\begin{tabular}{lcc}
\hline $\begin{array}{l}\text { Number of organ } \\
\text { systems affected }\end{array}$ & $\begin{array}{c}\text { Number of } \\
\text { chemicals }\end{array}$ & $\begin{array}{c}\text { \% of total } \\
\text { chemicals }\end{array}$ \\
\hline 1 & 32 & 30.8 \\
2 & 21 & 20.2 \\
3 & 17 & 16.3 \\
4 & 9 & 8.7 \\
5 & 5 & 4.8 \\
\hline 6 & 9 & 8.7 \\
7 & 5 & 4.8 \\
8 & 2 & 1.9 \\
9 & 1 & 1.0 \\
10 & 1 & 1.0 \\
11 & 2 & 1.9 \\
\hline Total & & \\
\hline
\end{tabular}

Data source: The EPA Integrated Risk Information System database, 1 January 2004. 


\section{Figure 1: Species used with assessed EPA chemicals lacking significant human data but} possessing animal data

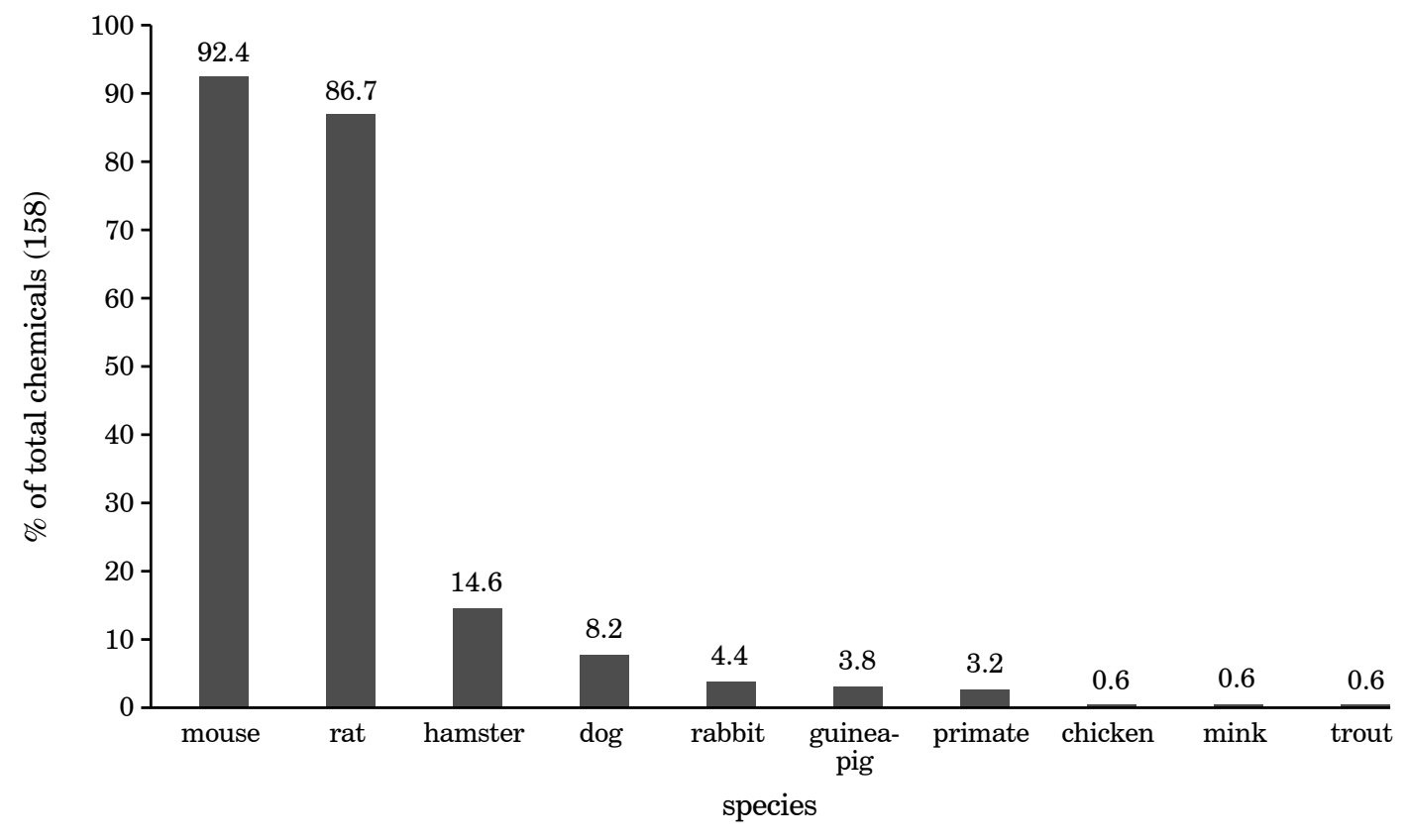

Data source: The EPA Integrated Risk Information System database, 1 January 2004.

(26.3\%). Other routes of major interest were drinking water $(21.1 \%)$ and inhalation $(17.9 \%)$.

\section{Organs affected}

Of the 160 chemicals, those considered probably not carcinogenic to humans were not, of course, known to exhibit significantly neoplastic lesions. For the 56 chemicals considered unclassifiable, it was frequently difficult to establish whether or not significant treatment-related results occurred. However, for the remaining 104 chemicals, considered probable or possible human carcinogens, up to 43 organs or organ systems were found to exhibit neoplastic lesions (Figure 3). However, given that only selected organ systems were examined by some investigators, and given that the location of several gastrointestinal and respiratory tract neoplasms were unspecified, the true prevalence of neoplastic lesions may have been even higher.

Up to 11 organ systems (in the case of 1,2-dibromoethane and $\mathrm{N}$-nitrosodiethylamine) were recorded as exhibiting neoplastic lesions for each chemical (Table 3), although most commonly one (30.8\%), two $(20.2 \%)$ or three $(16.3 \%)$ organ systems were affected. For some chemicals, unclear reporting may have resulted in duplication of results under multiple categories. For example, in a study of dietary aniline hydrochloride administered to rats, the investigators reported, among other tumours, statistically signifi- cant dose-related increases of haemangiosarcomas in the spleen, fibrosarcomas and sarcomas in the body cavity and spleen, and fibrosarcomas and sarcomas in multiple organs of the body cavity. The resulting classifications included connective tissue, peritoneal cavity, spleen and vascular system (7).

The organ systems most commonly affected (Figure 3) were the liver (66.3\%), the lung (31.7\%), and the kidney, skin and stomach (all 17.3\%). Differentiation between primary and metastatic tumours was often impossible, hence all tumours were included, even when (infrequently) identified as metastatic.

\section{Discussion}

\section{Species used}

As stated, the 158 applicable EPA chemicals were tested in up to seven species each, although two $(67.7 \%)$, one $(14.6 \%)$ or three $(11.4 \%)$ species were most commonly used (Table 1). Although at least 10 different animal species were used for these chemicals (Figure 1), the most commonly-used animals were mice $(92.4 \%)$ and rats $(86.7 \%)$.

\section{Discordance between mice and rats}

Intuitively, one might expect mice and rats to display similar carcinogen susceptibilities. However, 
Figure 2: Routes of administration for assessed EPA chemicals lacking significant human data but possessing animal data

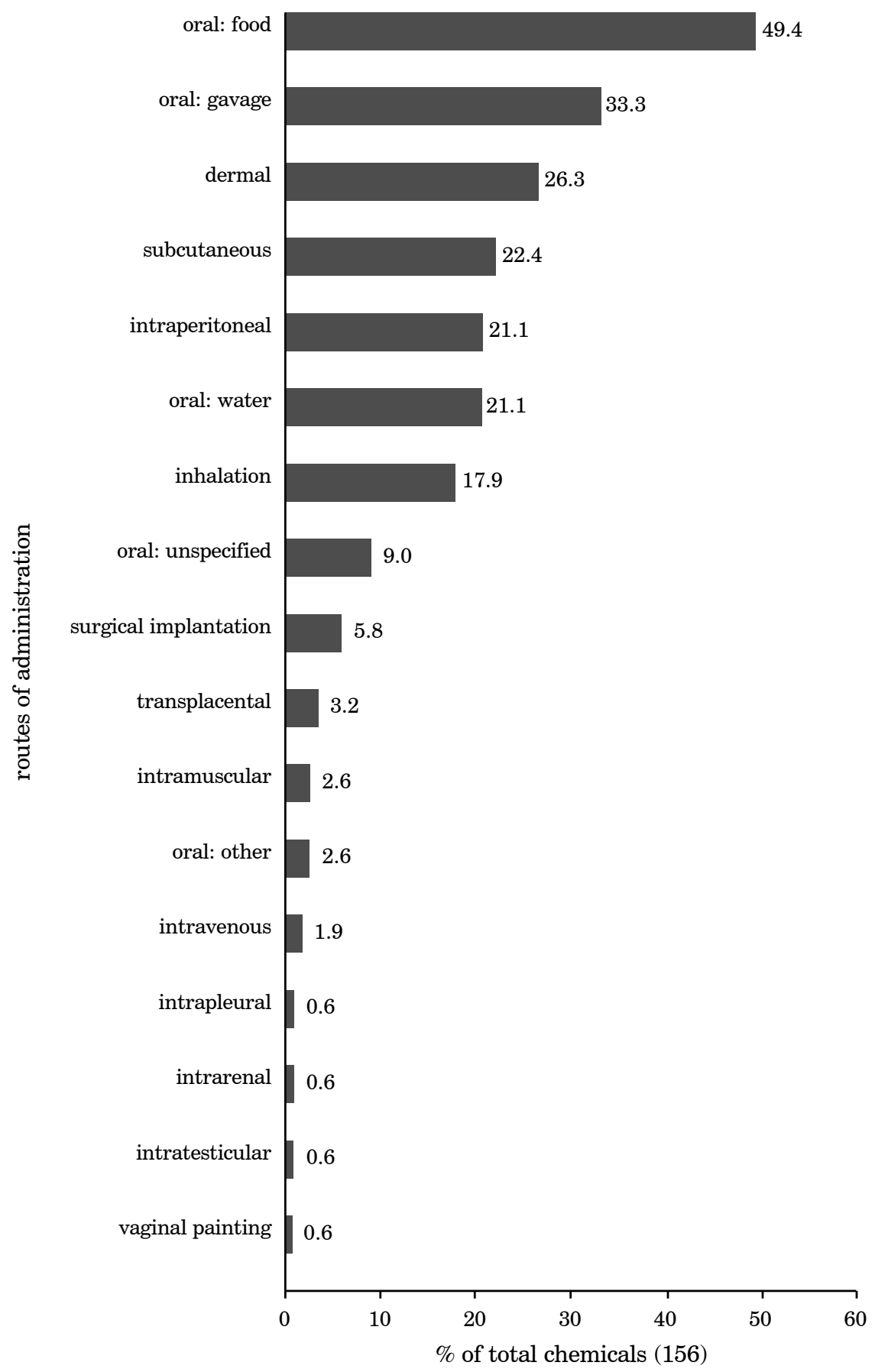

Data source: The EPA Integrated Risk Information System database, 1 January 2004.

while they do, for some chemicals, for others, they do not. For example, Hengstler et al. (8) described the striking variation in aflatoxin B1 carcinogenesis susceptibility between rats and mice, with mice refractory to dietary levels three orders of magnitude higher than those found to be carcinogenic in rats. Murine resistance to aflatoxin B1 results from an efficient conjugation with glutathione, catalysed by glutathione S-transferase $\mathrm{mYc}$, a biochemical mechanism apparently lacking in the rat.

In 2001, Gottman et al. (9) compared 121 replicated rodent carcinogenicity bioassays conducted 
Figure 3: Organ systems affected by chemicals assessed by the EPA to be probable (B2) or possible (C) human carcinogens

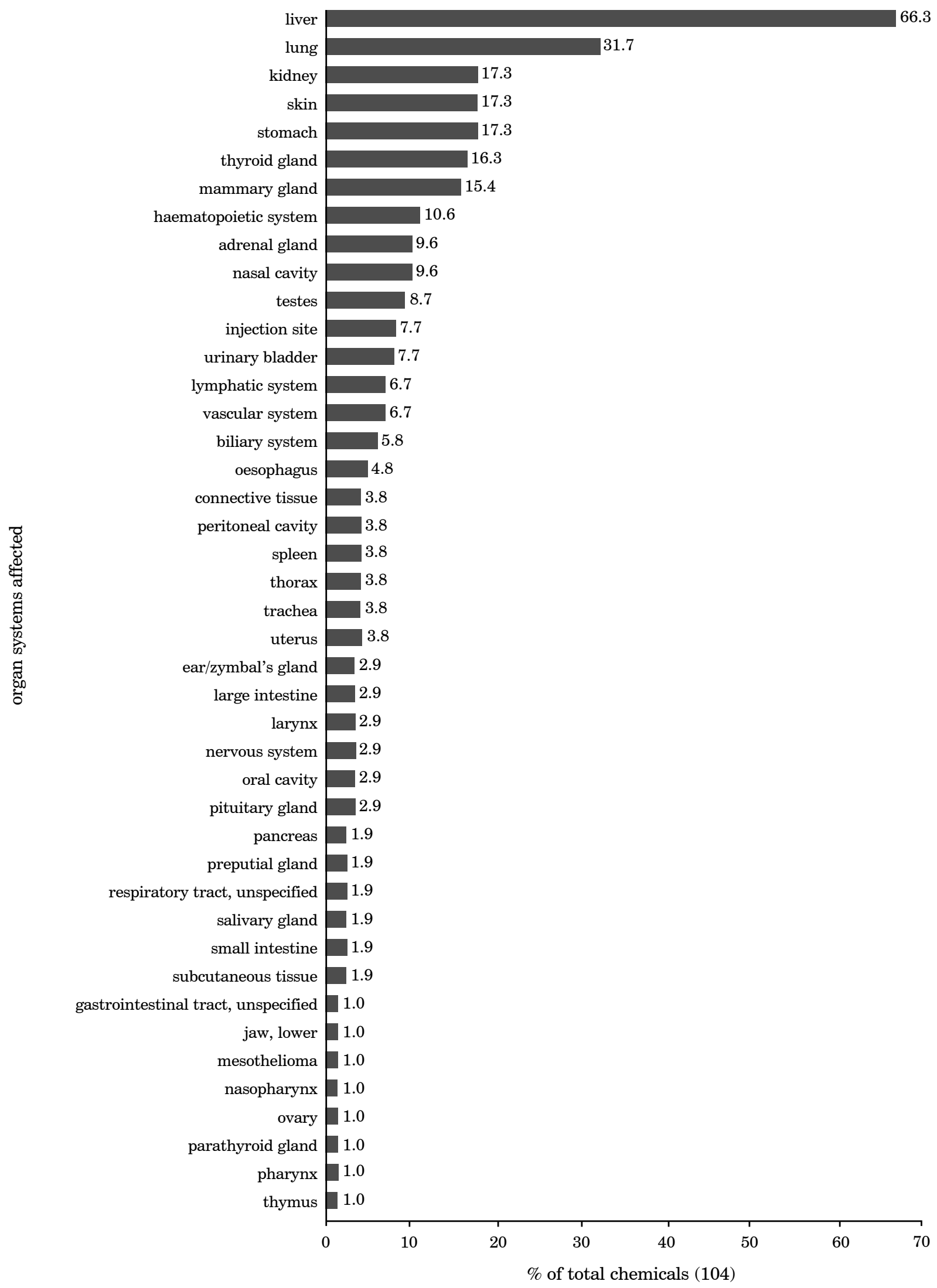

Data source: The EPA Integrated Risk Information System database, 1 January 2004. 
both within the US National Cancer Institute/ National Toxicology Program (NCI/NTP) and elsewhere, and published and recorded within the comprehensive Berkeley-based Carcinogenic Potency Database (CPDB). They found a reproducibility of only $57 \%$ overall for rodent carcinogenicity classifications between the NCI/NTP and studies from the general biomedical literature. This value did not improve substantially when additional biological information (species, strain, sex, target organs) was considered. Ettlin and Prentice (10), Fung et al. (11) and Johnson (12) have also demonstrated that bioassays involving strains other than those used by the NTP give discordant results.

Haseman (2) examined the long-term rodent studies of 385 substances recorded within the NCI/NTP database. After eliminating equivocal results, 207 chemicals were found to be carcinogenic in at least one sex-species group, yet only 56 of these $(27.1 \%)$ were carcinogenic in both male and female mice and rats. Male rats appeared to be more susceptible to tumourigenesis than females, but the situation was reversed in mice. Di Carlo (13) similarly found that of 61 chemicals causing cancer in mice or rats, only $13(21.3 \%)$ were carcinogenic in both male and female mice and rats. Di Carlo concluded that "it is painfully clear that carcinogenesis in the mouse cannot now be predicted from positive data obtained from the rat and vice versa."

\section{Discordance between rodents and primates}

The high carcinogenesis predisposition of rodents when compared to primates $(3,14)$ further complicates the extrapolation of results to humans. Detailed analyses of 25 rodent carcinogens tested in monkeys for up to 32 years by the NCI, revealed that half were not monkey carcinogens, in most cases despite strong evidence of carcinogenicity in rodents and/or humans (15). Another 26-year NCI study of model rodent carcinogens found that only urethane was carcinogenic in monkeys (16).

Numerous important differences between rodents and humans impact on predisposition to carcinogenesis. These differences between rats and humans include mean lifespan (2.5 versus 70 years), food consumption (50 versus $10 \mathrm{~g} / \mathrm{kg} /$ day), basal metabolic rate (109 versus $26 \mathrm{kcal} / \mathrm{kg} /$ day), anatomical differences (the forestomach, Zymbal's gland, Harderian gland, preputial gland and clitoral gland exist only in the rat), stomach $\mathrm{pH}$ (4-5 versus 1-2), and, very significantly, DNA excision repair rates (low versus high) (17).

Species differences in absorption, distribution, metabolism and elimination pathways or rates can all influence the carcinogenicity of a chemical. Since many carcinogens must be metabolised to reactive electrophiles to produce their carcinogenic effects, species differences in pathways or rates can affect carcinogenic metabolite activity levels (18). Examples of rodent carcinogens considered unclassifiable as to human carcinogenicity by the IARC, because their mechanisms of carcinogenesis are not present in humans (19-21), include $d$-limonene (rodent mechanism - renal tubular alpha2u-globulin mediated nephrotoxicity), saccharin and its salts, and melamine (rodent mechanism - urolithiasis predisposed by rodent urinary composition).

Indeed, it is remarkable that mice can develop very malignant tumours with multiple genetic alterations within 6-18 months, whereas aggressive tumours in humans or other primates may take many years to reach an equivalently life-threatening stage (22). Some $50 \%$ of all chemicals tested for carcinogenicity in mice or rats are positive in at least one experiment, with predisposition to carcinogenesis even higher in some commonly-used strains $(3,23)$. Holliday (24) suggested that the high predisposition of rodents to carcinogenesis when compared to humans, might be due to less-efficient DNA repair, poorer control of genetic stability, and/or altered control of gene expression. The high doses applied in rodent bioassays may also increase apparent carcinogenicity. The metabolic pathways and rates of activation and detoxification may become saturated at high tissue concentrations, resulting in differences in target tissue doses, altered tissue responses, and variations in the organs affected (18).

Of all of the species used, primates are the most biologically similar to humans. However, their lack of availability, high cost of procurement, housing and maintenance, and their lengthy life cycles, all serve to minimise their use (16). In contrast, the widespread availability, low costs of procurement, housing and maintenance, and the short life cycles of rodents, thereby facilitating lifetime exposure and developmental deformity studies, result in their predominant use in carcinogenicity bioassays. However, the largely logistical benefits of rodents do not qualify them scientifically as the test species of choice. In fact, the profound discordance of bioassay results between rodent species, strains and genders, and further, between rodents and human beings, means that it is profoundly difficult to make human carcinogenicity assessments on the basis of rodent bioassay data.

\section{Routes of administration}

\section{Carcinogenesis predisposition of stressful routes}

Studies on mice, rats, hamsters, monkeys, dogs, rabbits, birds, and even bats, have shown that basic nonor minimally-invasive procedures, such as handling or gavaging (administration of a test substance via 
an oesophageal tube), cause significant increases in stress indicators, including concentrations of glucose, corticosterone (a stress hormone), growth hormone, noradrenaline, prolactin, thyroid-stimulating hormone and triiodothyronine (25). Other blood measures, including packed cell volume, haemoglobin and plasma protein, also rise significantly (26). These stress-related responses generally occur with every exposure to such a stressor, and laboratory animals do not readily habituate to them (25).

Stress-related responses are particularly important in long-term carcinogenicity studies, in light of their frequent use of stressful routes of administration. Gavaging was used for $33.3 \%$ of the 156 applicable EPA chemicals under consideration, and dermal administration (requiring handling and restraint) for $26.3 \%$ (Figure 2). Other routes of administration used, which required handling and restraint as a minimum, were intramuscular, intraperitoneal, intrapleural, intrarenal, intratesticular, intravenous, oral (other than food or water, for example, via capsule or toothpaste additive), subcutaneous, surgical implantation and vaginal painting.

The stress-mediated hormonal changes and disruption of normal hormonal regulation that occur in response to such stressful stimuli, predispose animals to immunosuppression and increased susceptibility to virtually all pathologies, including neoplasia. Moynihan et al. (27) documented immunosuppression following handling, as evidenced by decreased immunoglobulin G levels in mice. Brenner et al. (28) documented more pulmonary metastases in 11 similarly-handled female mice than in non-handled controls, following intravenous challenge with tumour cells.

Tumour size can also be affected unpredictably by stressors, which complicates any assessments. Fifteen female mice handled daily (picked up by the tail and held gently in the palm for two minutes) on days one to five of tumour growth, had increases in tumour size compared to non-handled controls, while 13 mice similarly-handled on days one to seven did not (29).

Intra-laboratory and inter-laboratory variations in environmental conditions are also likely to result in varying hormonal disruptions and consequent immunosuppression. For example, animals are positioned in varying proximity to stressful stimuli such as doors, telephones, air-conditioning ducts and light bulbs. For some years, NTP rodent bioassay protocols used different shelves in the animal house for different treatment groups, potentially skewing the results (30).

To standardise and eliminate such variations in environmental conditions would be a very largescale and potentially expensive undertaking. Furthermore, it would not eliminate the variable but substantial stresses caused by handling and restraint, and the stressful routes of administration endemic to carcinogenicity bioassays, nor their inevitable effects on hormonal regulation, immune status, predisposition to carcinogenesis, and bioassay results.

\section{Route-to-route extrapolation}

Judgements frequently need to be made about the carcinogenicity of a chemical via a route of exposure different to that studied. For example, exposures of interest may sometimes be through the inhalation of a chemical tested primarily through feeding studies (31). Given that only $17.9 \%$ of the chemicals of interest were tested via inhalation, in contrast to the percentages tested via food $(49.4 \%)$, gavaging $(33.3 \%)$ or drinking water $(21.1 \%)$, such dilemmas hardly seem unlikely.

Quantitative extrapolation between routes of exposure is frequently problematic. Differences in rates of absorption and transport mechanisms between routes (for example, oral, inhalation, dermal) can be great. There is no generally applicable method for accounting for these differences in uptake processes, although confidence is strengthened when the tumour effects are observed at a site distant from the point of entry, or when absorption through the route of interest is similar to that via the tested route (31).

\section{Organs affected}

The 104 EPA chemicals considered to be probable or possible human carcinogens induced neoplasia in 43 different organ systems (Figure 3 ), with those most commonly affected being the liver $(66.3 \%)$, the lung $(31.7 \%)$, and the kidney, skin and stomach (all $17.3 \%)$. Up to 11 different organ systems were affected by each chemical, although most commonly one $(30.8 \%)$, two $(20.2 \%)$ or three $(16.3 \%)$ organ systems were affected (Table 3 ).

\section{Interspecies variation}

The wide variation in organ systems affected may have been exacerbated by the considerable variability of organ systems and species in response to many carcinogenic insults. Comparisons between mice, rats, hamsters and humans, for example, reveal that carcinogens are carcinogenic at the same site in another of these species no more than $50 \%$ of the time (3). Patterns of tumour incidence, whether spontaneous or in response to carcinogenic insults, differ dramatically between rodents and humans, and even between different rodent strains. High incidences of rare human tumours, such as those of the liver, pituitary and testis, occur spontaneously in various strains of mice or rats, whereas 
common human tumours, such as those of the prostate and colorectum, occur rarely, if ever, in rodents $(32-33)$.

\section{Dose-related toxicity}

In an attempt to interpret differing carcinogenic incidences across a large number of organ systems, investigators commonly assume that human carcinogenic risk is proportional to the number of organ systems affected, and that it increases when multiple sex-species groups are affected or when fatalities result. However these assumptions were undermined by Lois et al. (34), who found, in their analysis of around 4000 chronic carcinogenicity bioassays on 1050 chemicals, that carcinogens affecting multiple sex-species groups or causing fatalities are likely to be the same chemicals that affect multiple organ systems, and by Meijers et al. (35), who found that neoplastic lesions in multiple organ systems are more likely to be indicative of dose-related toxicity than true carcinogenicity.

Despite critics such as Monro and Davies (36), who point out that, since human carcinogens are amongst the most potent of rodent carcinogens it should be possible to detect them by using relatively low dose levels, carcinogenicity bioassays typically rely upon the maximum tolerated dose (MTD), as indicated by increasing toxicity-related effects, in order to maximise their sensitivity to carcinogens. However, prolonged exposure to high chemical doses can result in chronic irritation, cell death, and consequent cellular proliferation (mitogenesis). Sodium saccharin and related sodium and potassium salts, for example, produce urinary calculi, and consequently, cellular proliferation, regenerative hyperplasia and neoplasia, only at high doses (3, 37-38).

Animals have a broad range of physiological defences against general toxic insults, such as epithelial shedding and inducible enzymes, which commonly prove effective at environmentally relevant doses, but which may be overwhelmed at higher doses (3). When combined with insufficient rest intervals between doses for the effective operation of DNA and tissue repair mechanisms, the outcome can be an increased predisposition to mutagenesis and carcinogenesis. Lower doses, greater intervals between exposures, or shorter total periods of exposure, some or all of which represent a more realistic model of true environmental exposures for most potential carcinogens, might not result in carcinogenesis at all.

\section{Calorie-induced mitogenesis}

Reviews of both the experimental and epidemiological literature show a high correlation between increased cell division and carcinogenesis. Ad libitum feeding, as occurs in many carcinogenicity studies, can unnaturally elevate cell division (3), thereby increasing predisposition to carcinogenesis, with consequent increases in false positive rates.

Including indices of cell division for both test and control groups would facilitate the identification of groups with elevated cell division rates due to ad libitum feeding, thereby allowing the subsequently increased predisposition to carcinogenesis to be accounted for during the interpretation of the results. If necessary, feeding protocols could also be altered. Unfortunately, however, indices of cell division are not normally included or considered (3).

\section{False positive results}

It is consequently unsurprising that, for rodent carcinogens tested at the MTD and half the MTD, around half the organs in which tumours arise are only affected at the MTD (3). Clearly, chronic highdose carcinogenicity bioassays, in which physiological defences are overwhelmed and ad libitum feeding results in the unnatural elevation of cell division rates, are inherently predisposed towards false positive results. Such factors have been implicated as the cause of the poor human specificity of animal carcinogenicity studies, as revealed by other investigators (2-6) and ourselves (1).

\section{Conclusions}

Our survey of the 160 chemicals that had received EPA human carcinogenicity assessments primarily on the basis of their animal carcinogenicity data, revealed that a wide variety of species, predominantly rodents, were used, with a wide variety of routes of administration, and with effects on a particularly wide variety of organ systems.

The likely causes of the poor human specificity, and hence low predictivity, of rodent carcinogenicity bioassays revealed by other investigators (2-6) and ourselves (1), include: 1) the profound discordance of bioassay results between rodent species, strains and genders, and further, between rodents and humans; 2) the variable, yet substantial stresses, caused by handling and restraint and the stressful routes of administration common to carcinogenicity bioassays, with consequent effects on hormonal regulation, immune status and predisposition to carcinogenesis; 3 ) the differences in transport mechanisms and rates of absorption between test routes of administration and other important human routes of exposure; 4 ) the considerable variability of organ systems affected in response to carcinogenic insults, between and within species; and 5) the inherent predisposition of chronic high-dose bioassays toward false positive results, likely to be 
due to the overwhelming of physiological defences, and the unnatural elevation of cell division rates during ad libitum feeding. The EPA has acknowledged some of the difficulties on its IRIS website:

"In general IRIS values cannot be validly used to accurately predict the incidence of human disease or the type of effects that chemical exposures have on humans. This is due to the numerous uncertainties involved in risk assessment, including those associated with extrapolations from animal data to humans and from high experimental doses to lower environmental exposures. The organs affected and the type of adverse effect resulting from chemical exposure may differ between study animals and humans." (39).

Such factors result in the poor human specificity, and hence low predictivity, of animal carcinogenicity data, and render profoundly difficult any attempts to accurately extrapolate human carcinogenic hazards from animal data.

Fortunately, alternative carcinogenicity testing strategies exist, which have the potential to offer superior human specificity, as well as substantially reduced time-frames and greatly reduced demands on financial, personnel and animal resources. These alternatives are explored in detail in Knight et al. (40).

\section{Acknowledgement}

This research was partly funded by the Physicians Committee for Responsible Medicine, Washington DC, USA.

Received 6.8.05; received in final form 18.12.05; accepted for publication 19.12.05.

\section{References}

1. Knight, A., Bailey, J. \& Balcombe, J. (2006). Animal carcinogenicity tests: 1 . poor human predictivity. ATLA 34, 19-27.

2. Haseman, K. (2000). Using the NTP database to assess the value of rodent carcinogenicity studies for determining human cancer risk. Drug Metabolism Reviews 32, 169-186.

3. Gold, L.S., Slone, T.H. \& Ames, B.N. (1998). What do animal cancer tests tell us about human cancer risk? Overview of analyses of the carcinogenic potency database. Drug Metabolism Reviews 30, 359-404.

4. Ashby, J. \& Purchase, I.F. (1993). Will all chemicals be carcinogenic to rodents when adequately evaluated? Carcinogenesis 8, 489-495.

5. Rall, D.P. (2000). Laboratory animal tests and human cancer. Drug Metabolism Reviews 2, $119-128$.

6. International Agency for Research on Cancer (1972-1992). IARC Monographs on the Evaluation of
Carcinogenic Risks to Humans. Volumes 1-55. Lyon, France: International Agency for Research on Cancer.

7. National Cancer Institute (1978). Bioassay for Aniline Hydrochloride for Possible Carcinogenicity. CAS No. 142-04-1. ITS Carcinogenesis Technical Report Series No. 130. Department of Health, Education and Welfare (DHEW) Publication No. NIH 78-1385. 113pp. Bethesda, MD, USA: DHEW.

8. Hengstler, J.G., Van der Burg, B., Steinberg, P. \& Oesch, F. (1999). Interspecies differences in cancer susceptibility and toxicity. Drug Metabolism Reviews 31, 917-970.

9. Gottmann, E., Kramer, S., Pfahringer, B. \& Helma, C. (2001). Data quality in predictive toxicology: reproducibility of rodent carcinogenicity experiments. Environmental Health Perspectives 109, 509-514.

10. Ettlin, R.A. \& Prentice, D.E. (2002). Unexpected tumour findings in lifetime rodent bioassay studies — what to do. Toxicology Letters 128, 17-33.

11. Fung, V.A., Barrett, J.C. \& Huff, J. (1995). The carcinogenesis bioassay in perspective: application in identifying human cancer hazards. Environmental Health Perspectives 103, 680-683.

12. Johnson, F.M. (1999). Carcinogenic chemical-response "fingerprint" for male F344 rats exposed to a series of 195 chemicals: implications for predicting carcinogens with transgenic models. Human Ecology and Risk Assessment 5, 427-443.

13. Di Carlo, F.J. (1984). Carcinogenesis bioassay data: correlation by species and sex. Drug Metabolism Reviews 15, 409-413.

14. Goodman, J.I. (2001). A perspective on current and future uses of alternative models for carcinogenicity testing. Toxicologic Pathology 29, Suppl. 1, 173-176.

15. Gold, L.S., Manley, N.B., Slone, T.H. \& Rohrbach, L. (1999). Supplement to the Carcinogenic Potency Database (CPDB): results of animal bioassays published in the general literature in 1993 to 1994 and by the National Toxicology Program in 1995 to 1996. Environmental Health Perspectives 107, Suppl. 4, 527-600.

16. Schoeffner, D.J. \& Thorgeirsson, U.P. (2000). Susceptibility of nonhuman primates to carcinogens of human relevance. In Vivo 14, 149-156.

17. Monro, A. \& Mordenti, J. (1995). Expression of exposure in negative carcinogenicity studies: dose/body weight, dose/body surface area, or plasma concentrations? Toxicologic Pathology 23, 187-198.

18. Dybing, E. \& Huitfeldt, H.S. (1992). Species differences in carcinogen metabolism and interspecies extrapolation. In Mechanisms of Carcinogenesis in Risk Identification (ed. H. Vainio, P.N. Magee, D.B. McGreger \& A.J. McMichael), pp. 501-522. Lyon, France: International Agency for Research on Cancer.

19. International Agency for Research on Cancer (1999). IARC Monographs Programme on the Evaluation of Carcinogenic Risks to Humans. Vol. 73: Some Chemicals that Cause Tumours of the Kidney or Urinary Bladder in Rodents, and Some Other Substances. 674pp. Lyon, France: International Agency for Research on Cancer. Website http://monographs. iarc.fr (Accessed 20.01.04).

20. Hard, G.C. \& Whysner, J. (1994). Risk assessment of d-limonene: an example of male rat-specific renal tumorigens. Critical Reviews in Toxicology 24, 231-254.

21. Environmental Protection Agency (1991). Report of the EPA Peer Review Workshop on Alpha2u-globulin: Association with Renal Toxicity and Neoplasia 
in the Male Rat. 93pp. Washington DC, USA: Environmental Protection Agency.

22. Balmain, A. \& Harris, C.C. (2000). Carcinogenesis in mouse and human cells: parallels and paradoxes. Carcinogenesis 21, 371-377.

23. Gold, L.S. \& Zeiger, E., eds (2000). Handbook of Carcinogenic Potency and Genotoxicity Databases. 770pp. Boca Raton, FL, USA: CRC Press.

24. Holliday, R. (1996). Neoplastic transformation: the contrasting stability of human and mouse cells. Cancer Surveys 28, 103-115.

25. Balcombe, J.P., Barnard, N. \& Sandusky, C. (2004). Laboratory routines cause animal stress. Contemporary Topics in Laboratory Animal Science 43, 42-51.

26. Gärtner, K., Büttner, D., Döhler, K., Friedel, R., Lindena, J. \& Trautschold, I. (1980). Stress response of rats to handling and experimental procedures. Laboratory Animal 14, 267-274.

27. Moynihan, J., Brenner, G., Koota, D., Breneman, S., Cohen, N. \& Ader, R. (1990). The effects of handling on antibody production, mitogen responses, spleen cell number, and lymphocyte subpopulations. Life Sciences 46, 1937-1944.

28. Brenner, G.J., Cohen, N., Ader, R. \& Moynihan, J.A. (1990). Increased pulmonary metastases and natural killer cell activity in mice following handling. Life Sciences 47, 1813-1819.

29. Aarstad, H.J. \& Seljelid, R. (1992). Effects of stress on the growth of a fibrosarcoma in nu/nu and conventional mice. Scandinavian Journal of Immunology 35, 209-215.

30. Young, S.S. (1989). What is the proper experimental unit for long-term rodent studies? An examination of the NTP benzyl acetate study. Toxicology 54, 233-239.

31. Anon. (1999). Guidelines for Carcinogen Risk Assess- ment. 211pp. Washington DC, USA: Risk Assessment Forum, Environmental Protection Agency.

32. Monro, A. (1993). How useful are chronic (life-span) toxicology studies in rodents in identifying pharmaceuticals that pose a carcinogenic risk to humans? Adverse Drug Reactions and Toxicological Reviews 12, 5-34.

33. Monro, A. (1993). The paradoxical lack of interspecies correlations between plasma concentrations and chemical carcinogenicity. Regulatory Toxicology and Pharmacology 18, 115-135.

34. Lois, S.W., Slone, T.H., Manley, N.B., Garfinkel, G.B., Hudes, E.S., Rohrbach, L. \& Ames, B. (1991). The Carcinogenic Potency Database: analyses of 4000 chronic animal cancer experiments published in the general literature and by the U.S. National Cancer Institute/National Toxicology Program. Environmental Health Perspectives 96, 11-15.

35. Meijers, J.M., Swaen, G.M. \& Bloemen, L.J. (1997). The predictive value of animal data in human cancer risk assessment. Regulatory Toxicology and Pharmacology 25, 94-102.

36. Monro, A. \& Davies, T.S. (1993). High dose levels are not necessary in rodent studies to detect human carcinogens. Cancer Letters 75, 183-194.

37. Cohen, S.M. \& Lawson, T.A. (1995). Rodent bladder tumors do not always predict for humans. Cancer Letters 93, 9-16.

38. Cohen, S.M. (1995). Human relevance of animal carcinogenicity studies. Regulatory Toxicology and Pharmacology 21, 75-80.

39. Anon. (2006). IRIS limitations. Website www.epa. gov/IRIS/limits.htm (Accessed 05.02.06)

40. Knight, A., Bailey, J. \& Balcombe, J. (2006). Animal carcinogenicity tests: 3 . alternatives to the bioassay. ATLA 34, 39-48. 